\title{
Search for dark forces in flavor experiments
}

\section{Thomas Czank*}

for the Belle Collaboration

Tohoku University, Sendai

E-mail: thomaseepx.phys.tohoku.ac.jp

The search for dark forces in flavor experiments is motivated by the lack of direct detection results, that were initially fueled by astronomical observations, such as gravitational lensing and CMB oscillations involving the Galaxy rotation problem, in which visible elements farther from the Galaxy center rotate with the same speed or faster than those close to the center. Besides the direct detection attempts, the long proposed model of WIMP (Weakly Interacting Massive Particle) as a dark matter candidate has not been fruitful yet. These shortcomings have allowed for new trial models such as portal interactions through new mediators that mix with SM ones, the dark photon, the right handed neutrino or the axiom, to be probed in the range of flavor experiments.

The 15th International Conference on Flavor Physics \& CP Violation

5-9 June 2017

Prague, Czech Republic

${ }^{*}$ Speaker. 


\section{Introduction}

The original motivations for dark matter models are closely related to astronomical observations. One of the first evidences purposing to dark matter is the Galaxy rotation problem, the speed of visible astronomical objects farther from their Galaxy center was expected to be smaller than the speed of those close to the Galaxy center, since the amount of visible objects at edges of galaxies is also smaller than at the center, but the observed result was different.
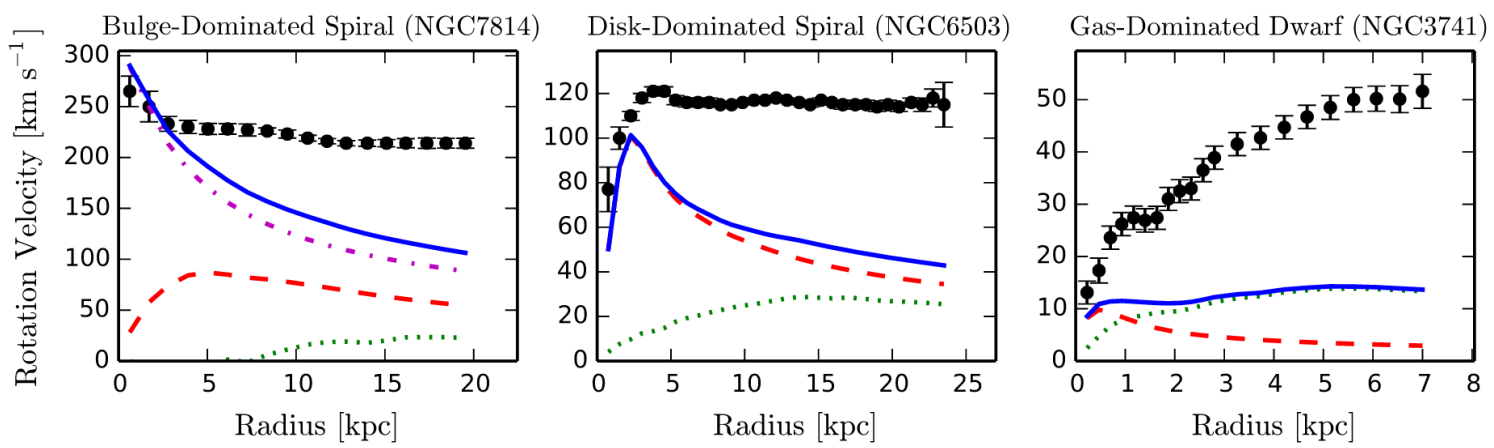

Figure 1: $\underline{\text { Black dots }}$ are the observed rotation curves, dotted lines are gas components, dashed lines are stellar disk, dot and dashed lines are the Bulge and the continuous line is the visible components together [1]

This result allow for the proposal that there is extra invisible matter, which doesn't interact with the electromagnetic force carrier, the photon, therefore, dark matter. Other evidences are the CMB anisotropy spectrum [2]. In which the Cosmic Microwave Background's (CMB) photons are scattered accross the universe in a very uniform way, yet there are still perturbations, a temperature difference distribution dependent on the angular measurement position, from probes, such as the Wilkinson Microwave Anisotropy Probe (WMAP), the CMB Anisotropy distribution is related to Dark Matter distribution. Or gravitational lensing caused by dark matter [3].

The initial model for this large amount of invisible mass permeating the universe was a Weakly Interacting Massive Particle (WIMP), which only interact gravitationally or weakly, with some models that fit those requirements, such as the neutralino, the little higgs or the gravitno [4].

Many experiments have been searching for the WIMP candidates through nuclear recoil, using heavy and very stable elements isolated from most sources of radiation.

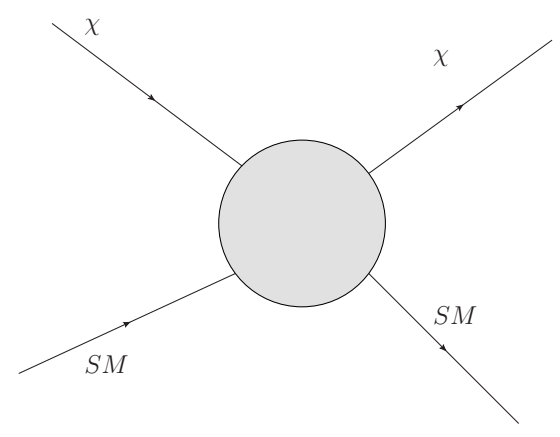

Figure 2: Direct detection diagram for a wimp candidate particle $\chi$ bumping on a SM atom nucleus making it recoil 
This kind of experiment has been conducted by various collaborations around the world and even though no signal was found some mass ranges have been constrained.

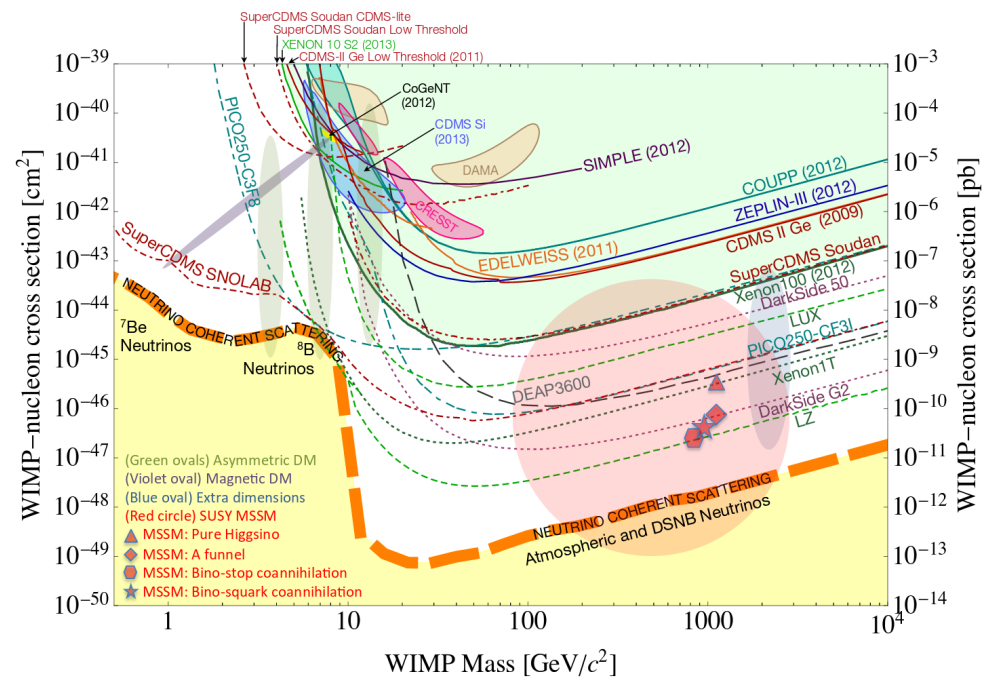

Figure 3: Plot with many WIMP search experiments and other conditions that constrain the WIMP mass ranges [5]

In Fig. 3 the lower shaded region in light yellow under the dashed orange line is constrained due to the Neutrino Coherent Scattering of Atmospheric and Diffuse Supernova Neutrinos Background (DSNB) that can cause neutrino-induced recoil events which would lower the chance of detecting WIMP induced recoil events [6]. Apart from this region there is also a light red shaded circle that is covered by Minimal Super Symmetric Models (MSSMs), a light blue oval shaded region due to extra dimensional models, a light green oval shaded region due to the Asymmetric Dark Matter (DM) models and finally a light violet oval shaded region due to Magnetic DM models. Even though with all this restrictions the upper left region still has very few constraints, this region of mass range lower $10 \mathrm{GeV}$ is probable by many flavor experiments.

Another evidence that point out to lower masses models of a Dark Sector is the observation of increasing positron fraction with increasing energy at Cosmic ray detection experiments at Fig. 4.

This positron fraction increase with the cosmic rays energy is not expected from the SM. An antiproton fraction increase is not observed, therefore, explanations based on the annihilation of dark matter particles producing a Dark Photon, $A^{\prime}$ a secluded $U(1)_{D}$ boson, have their mass limited at $<2 \mathrm{GeV}$. This secluded boson would then mix with SM bosons, such as $\gamma$ or $Z$ finally decaying into SM particles. Regarding the range $>10 \mathrm{GeV}$, this positron fraction increase could be related to the heavy neutralino that could decay to leptons at even higher energies. As mentioned earlier many flavor experiments are highly sensible to the dark photon in this region, $1 \mathrm{MeV} \sim 10 \mathrm{GeV}$.

\section{Dark Sector Models}

The Dark Sector is the name given to a collection of particles, DM included, that do not interact by any SM gauge boson directly [8], however they might interact with SM models via portals, consisting of secluded gauge bosons that mix with the SM ones or new undiscovered particles such 


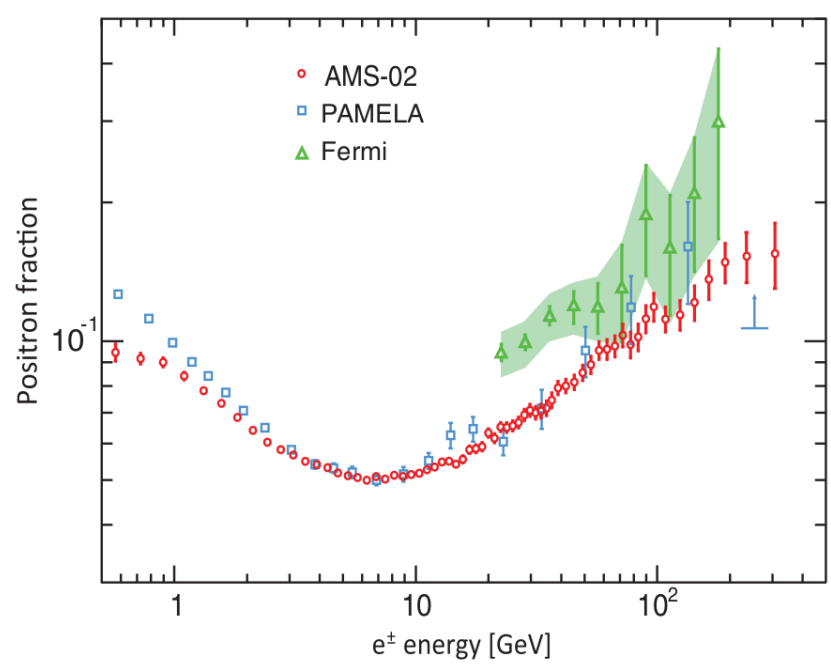

Figure 4: Plot with different experiments measurements of the positron fraction anomaly at Cosmic Rays [7]

as the axiom or the right handed neutrino. These are alternate models that assume no direct SM interaction, differently from WIMPs.

Portal interactions can be listed as follows:

- Neutrino Portal in which Dark Matter is considered only to interact with SM via neutrinos or a charged Higgs [9]. $\mathscr{L}_{v} \propto y_{n} L H N$, where $y_{n}$ is the neutrino Yukawa coupling, $L$ is a lepton doublet, $H$ is the SM Higgs doublet and $N$ is the right handed Neutrino.

- Vector Portal in which Dark matter interacts with SM particles via a Dark Photon that mixes with the SM photon allowing decays into SM particles. Alternative vector portals propose other dark gauge bosons that couples specifically to leptons or hadrons. This portal will be discussed further here in detriment of the others. $\mathscr{L}_{A^{\prime}} \propto-\frac{\varepsilon}{2 \cos \theta_{W}} B_{\mu v} Z^{\prime \mu v}$, where $\varepsilon$ is the mixing parameter between SM and Dark photons, $\cos \theta_{W}$ is the weak mixing angle, $B_{\mu v}$ hypercharge field strength tensor and $Z^{\prime \mu v}$ is the Dark Photon field strength tensor.
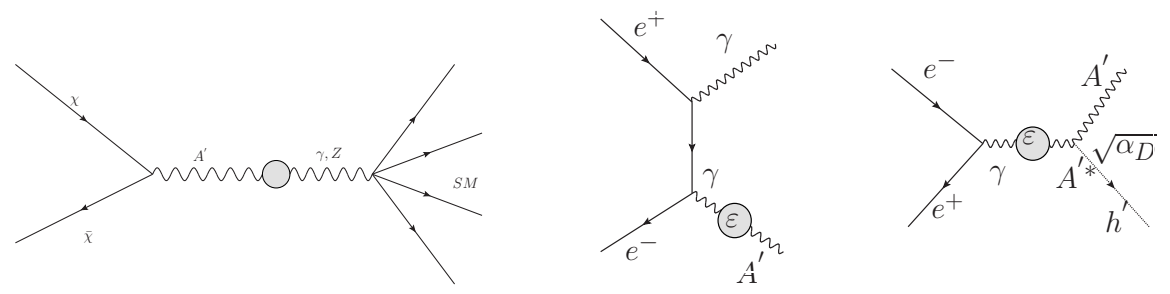

Figure 5: Different Feynman Diagrams (leftmost) in which dark matter annihilates into SM matter. (center) A Bahba scattering generates a dark photon, $A^{\prime}$, by the mixing factor, $\varepsilon$, with the SM photon, $\gamma$. (rightmost) An electron positron annihilation resulting into a dark photon mixing with the dark higgs $h^{\prime}$ by $\sqrt{\alpha_{D}}$.

- minimal kinetically mixed dark photon

$$
\mathscr{L}_{\text {int }_{A}^{\prime}} \propto \frac{1}{2} \frac{\varepsilon}{\cos \theta_{W}} F^{\mu v} Z_{\mu v}^{\prime}, \varepsilon=\frac{\alpha^{\prime}}{\alpha}
$$


where $\alpha^{\prime}$ is the dark coupling constant and $\alpha$ is the SM one.

- SM gauge group $S U(3)_{c} \times S U(2)_{L} \times U(1)_{Y}$

- Dark vector boson group $U(1)_{D}$

- Higgs Portal in which Dark matter candidates might annihilate into SM via Higgs, [10]. Or alternatively via a mixing of SM and secluded higgs models interacting with other secluded gauge bosons, such as the dark photon. $\mathscr{L}_{h^{\prime}} \propto\left(\mu \phi+\lambda \phi^{2}\right) H^{\dagger} H, \mu$ and $\lambda$ are constants whereas $\phi$ is a scalar.

- Axion Portal, originally a hypothetical particle that would barely interact with SM electromagnetic field mediator [11]. Alternatively it could interact with DM candidates via a SM photon and a Dark photon mixing [12]. $\mathscr{L}_{a} \propto \frac{a}{f_{a}} F_{\mu \nu} \tilde{F}^{\mu v}, f_{a}$ mass scale factor, $a$ is the axion pseudo-scalar, $F_{\mu v}\left(\tilde{F}^{\mu v}\right)$ is the dual field strength tensor of the SM photon field.

\section{Flavor Experiments}

Flavor experiments are usually defined as collider experiments that operate in flavor eigenstates resonances energy range, such as Belle and BaBar that are B-factories, producing $b$ flavored eigenstate resonance, a $\Upsilon(4 S)$ that then decays into two $B$ mesons. Other than B-factories there are also Charm factories, such as BES III, $\phi$-factories such as KLOE 2 , as well as others.
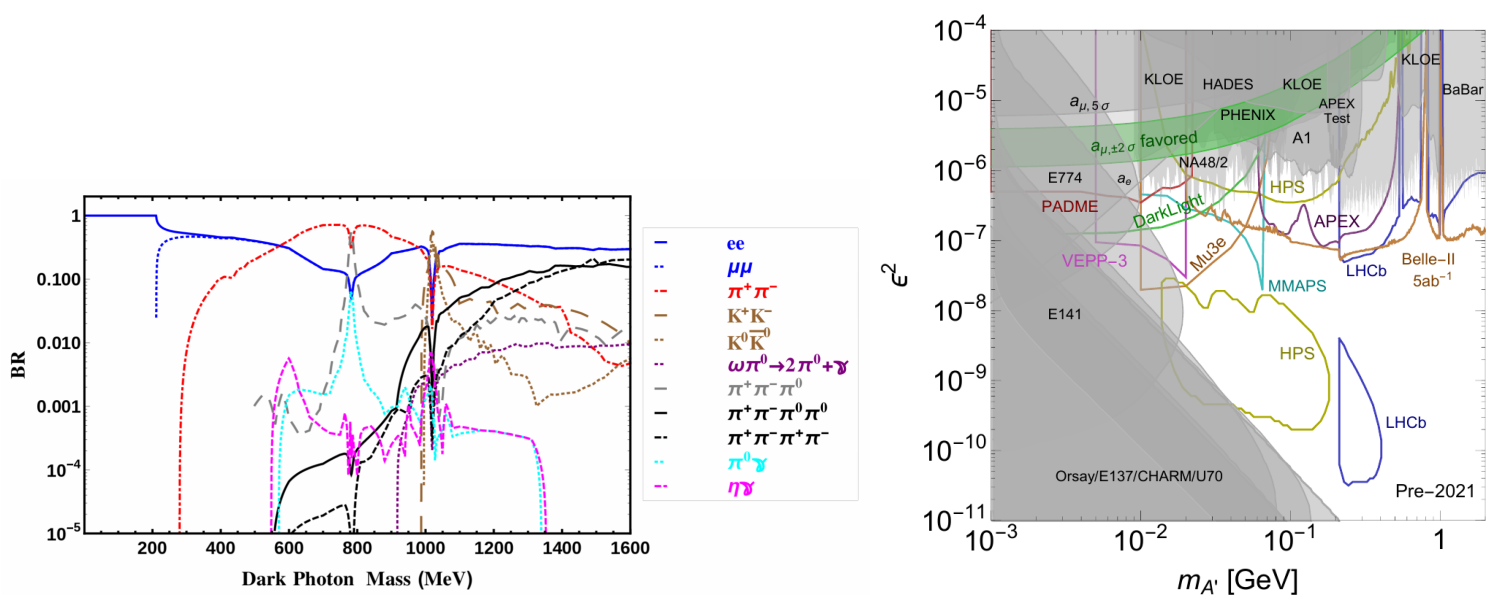

Figure 6: Left figure is the Branching Ratio for the Dark Photon, the right one shows the shaded regions corresponding to the mixing parameter by dark photon mass that have been constrained, the non shaded regions bordered by colorful lines are displaying future experiments [8]

On Table 1 most flavor experiments fall in the $e^{+} e^{-}$collider class, and usually their detection strategy is a bump hunt, where by checking the correspondent dark photon branching ratio final state, eg: $\ell^{+} \ell^{-}$, to the operation energy range of the flavor experiment it is possible to look for an enhancement over the continuous spectrum of the final state from $e^{+} e^{-} \rightarrow \gamma A^{\prime}$, eg: a distinguished peak over the invariant mass of a lepton pair.

As examples for the dark forces searches in flavor experiments the shaded rows will be given as examples, BES-III in orange is one of the newest results, March 2017, on the dark photon search. 
Table 1: Summary of the kind of dark photon searches that each class of experiment can perform, the first line shaded in pink corresponds to most Flavor experiments searches [8]

\begin{tabular}{|l|l|l|}
\hline Experiment Class & Typical Production Modes & Detection \\
\hline$e^{+} e^{-}$collider & $e^{+} e^{-} \rightarrow \gamma A^{\prime}$ & bump hunt \\
Electron fixed-target & $e^{-} Z \rightarrow e^{-} Z A^{\prime}$ & DM scatter or bump hunt \\
Hadron collider & $p p \rightarrow(\mathrm{jet} / \gamma) A^{\prime}$ & bump hunt or Drell-Yan \\
Positron fixed-target & $e^{+} e^{-} \rightarrow \gamma A^{\prime}$ & bump hunt \\
Proton fixed-target & $\pi^{0} / \eta / \eta^{\prime} \rightarrow \gamma A^{\prime}, q \bar{q} \rightarrow A^{\prime}, p Z \rightarrow p Z A^{\prime}$ & DM scatter or Drell-Yan \\
\hline
\end{tabular}

Table 2: Summary of the kind of dark photon searches that each class of experiment can perform, the first line shaded in pink corresponds to most Flavor experiments searches [8]

\begin{tabular}{|c|c|c|c|c|c|c|c|c|c|}
\hline Exp & Lab & Product & Detect & $\mathrm{M}(\mathrm{MeV})$ & Res $(\mathrm{MeV})$ & Beam & EBeam $(\mathrm{GeV})$ & Ibeam/Lim & Run \\
\hline \hline APEX & JLab & e-brem & $\ell^{+} \ell^{-}$ & $65-600$ & $0.5 \%$ & $e^{-}$ & $1.1-4.5$ & $150 \mu \mathrm{A}$ & $2010(18)$ \\
\hline A1 & Mainz & e-brem & $e^{+} e^{-}$ & $40-300$ & $?$ & $e^{-}$ & $0.2-0.9$ & $140 \mu \mathrm{A}$ & 2011 \\
\hline HPS & JLab & e-brem & $e^{+} e^{-}$ & $20-200$ & $1-2 \%$ & $e^{-}$ & $1-6$ & $50-500 \mathrm{nA}$ & $2015(18)$ \\
\hline DarkLight & JLab & e-brem & $e^{+} e^{-}$ & $<80$ & $?$ & $e^{-}$ & 0.1 & $10 \mathrm{~mA}$ & 2020 \\
\hline MAGIX & Mainz & e-brem & $e^{+} e^{-}$ & $10-60$ & $?$ & $e^{-}$ & 0.155 & $1 \mathrm{~mA}$ & 2020 \\
\hline NA64 & CERN & e-brem & $e^{+} e^{-}$ & $1-50$ & $?$ & $e^{-}$ & 100 & $2 \times 10^{11} \mathrm{EOT} / \mathrm{yr}$ & $2017(22)$ \\
\hline Super-HPS & SLAC & e-brem & visible & $<500$ & $?$ & $e^{-}$ & $4-8$ & $1 \mu \mathrm{A}$ & $?$ \\
\hline \hline VEPP3 & Budker & annih & invis & $5-22$ & 1 & $e^{+}$ & 0.500 & $10^{33} \mathrm{~cm}^{-2} / \mathrm{s}$ & 2019 \\
\hline PADME & Frascati & annih & invis & $1-24$ & $2-5$ & $e^{+}$ & 0.550 & $\leq 10^{14} e^{+} \mathrm{OT} / \mathrm{y}$ & 2018 \\
\hline MMAPS & Cornell & annih & invis & $20-78$ & $1-6$ & $e^{+}$ & 6.0 & $10^{34} \mathrm{~cm}^{-2} / \mathrm{s}$ & $?$ \\
\hline \hline BES-III & BEPC II & several & vis/in & $\lesssim 5 \mathrm{GeV}$ & 1 & $e^{+} e^{-}$ & $\leq 4.63$ & $10^{33} \mathrm{~cm}^{-2} / \mathrm{s}$ & 2008 \\
\hline BaBar & PEP II & several & vis/in & $\lesssim 12 \mathrm{GeV}$ & $1-5$ & $e^{+} e^{-}$ & $9 \times 3.1$ & $3 \times 10^{33} \mathrm{~cm}^{-2} / \mathrm{s}$ & 2008 \\
\hline KLOE 2 & Frascati & several & vis/in & $<1.1 \mathrm{GeV}$ & 1.5 & $e^{+} e^{-}$ & 0.51 & $2 \times 10^{32} \mathrm{~cm}^{-2} / \mathrm{s}$ & 2014 \\
\hline BELLE & KEKB & several & vis/in & $\lesssim 10 \mathrm{GeV}$ & $1-5$ & $e^{+} e^{-}$ & $8 \times 3.5$ & $2 \times 10^{34} \mathrm{~cm}^{-2} / \mathrm{s}$ & 2008 \\
\hline Belle II & SuperKEK & several & vis/in & $\lesssim 10 \mathrm{GeV}$ & $1-5$ & $e^{+} e^{-}$ & $7 \times 4$ & $1 \sim 10 \mathrm{ab} / 1$ & 2018 \\
\hline \hline SeaQuest & FNAL & several & $\mu^{+} \mu^{-}$ & $\lesssim 10 \mathrm{GeV}$ & $3-6 \%$ & $\mathrm{p}$ & 120 & $10^{18} \mathrm{POT} / \mathrm{y}$ & $2017(20)$ \\
\hline SHIP & CERN & several & visible & $\lesssim 10 \mathrm{GeV}$ & $1-2$ & $\mathrm{p}$ & 400 & $2 \times 10^{20} \mathrm{POT} / 5 \mathrm{y}$ & 2026 \\
\hline LHCb & CERN & several & $\ell^{+} \ell^{-}$ & $\lesssim 40 \mathrm{GeV}$ & $\sim 4$ & $\mathrm{pp}$ & 6500 & $\sim 10 \mathrm{fb}^{-1} / y$ & $2010(15)$ \\
\hline
\end{tabular}

The yellow shaded BaBar and BELLE are concluded experiments with data still to be analyzed, finally the green shaded KLOE 2 and Belle II are experiments that are result of recent upgrades and if have not yet started data taking will start soon.

\section{Dark forces searches}

\subsection{BES III Search}

The Beijing Electron Spectrometer (BES III) is located in the double ring $e^{+} e^{-}$Beijing Electron Positron Collider (BEPCII) which is a symmetric collider operating in the range $2<\sqrt{s}<4.63$ $\mathrm{GeV}$, a $\tau$-Charm factory with an integrated luminosity of $10.3 \mathrm{fb}^{-1}$. More detailed information on 
BES III design at [13]. Using a data set of $2.93 \mathrm{fb}^{-1}$ taken at $3.773 \mathrm{GeV}$ a search for an extra $U(1)$ gauge boson, or dark photon, was conducted looking for a bump in the lepton-pair invariant mass distribution $e^{+} e^{-} \rightarrow \ell^{+} \ell^{-} \gamma_{\mathrm{ISR}}$ where $\ell=\mu$ or $e$ [14]. This search is mainly motivated by astrophysical observations [7] hinting for a dark photon at the $\mathrm{MeV} / c^{2} \sim \mathrm{GeV} / c^{2}$ mass scale, besides that the dark photon could also be related to the 3 to $4 \sigma$ deviation between the $(g-2)_{\mu}$ measurement and its SM prediction [15] could be due to the dark photon muon coupling.
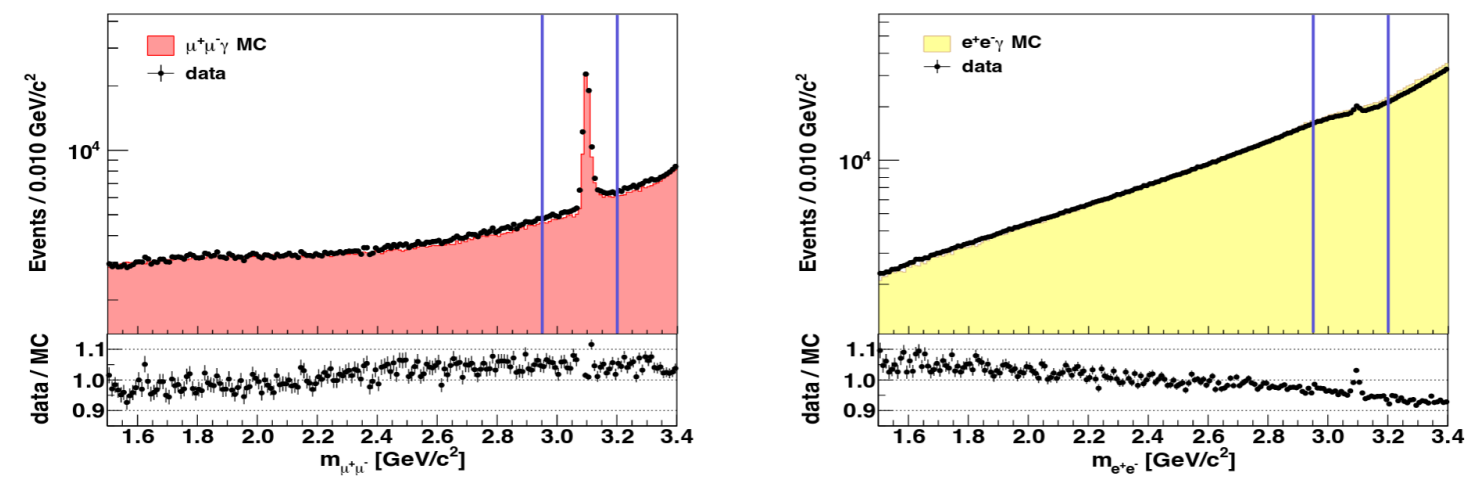

Figure 7: Invariant mass distribution for different lepton pairs, $m_{\mu^{+} \mu^{-}}$on the left and $m_{e^{+} e^{-}}$on the right, the blue lines show the $J / \psi$ excluded resonance region from the analysis [14]

Looking at the lepton pairs invariant mass distributions below, Fig. 7, no enhancement due to a possible dark photon decay is found. After the search a BES III exclusion limit is drawn, and it overlaps a previous dark photon search at BaBar, this is probably due to BaBar's bigger integrated luminosity and single photon trigger, allowing BaBar to get a clearer signal, which BES III doesn't have.

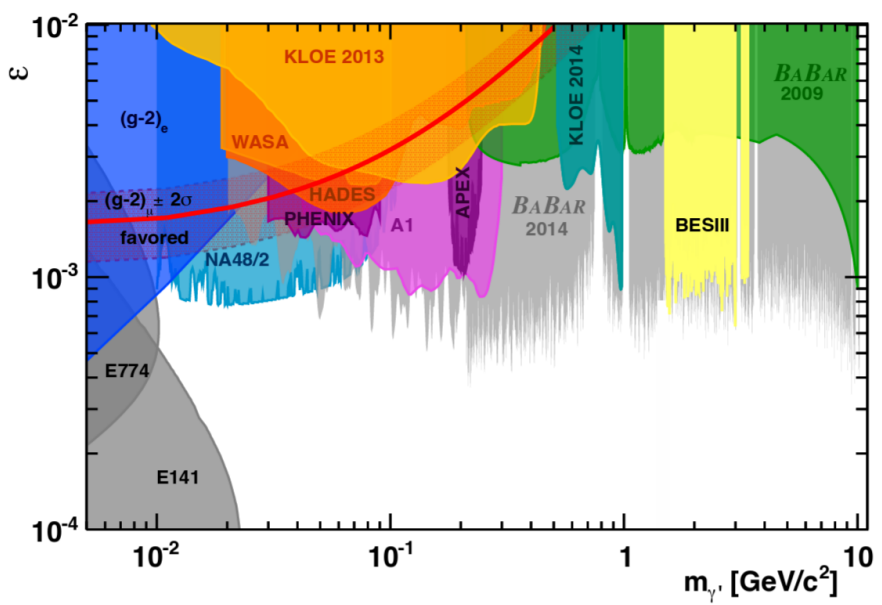

Figure 8: Exclusion limit for the dark photon, SM photon mixing parameter $\varepsilon$ from many experiments and BES III on light yellow overlapping BaBar gray excluded region [14]

\subsection{BELLE quarkphilic dark gauge boson search}

BELLE was a detector based at the KEKB asymmetric $e^{-} e^{+}$collider operating as a B-factory, for more information on the BELLE design [16] . A search for the quark coupled dark gauge $U^{\prime}$ 
was conducted using $976 \mathrm{fb}^{-1}$ of BELLE data at the $290 \sim 520 \mathrm{MeV} / c^{2}$ range in the decay chain $D^{*+} \rightarrow D^{0} \pi^{+}, D^{0} \rightarrow K_{S}^{0} \eta, \eta \rightarrow U^{\prime} \gamma, U^{\prime} \rightarrow \pi^{+} \pi^{-}$[17]. The $U^{\prime}$ search is a different one compared to the dark photon cases but a similar analysis strategy is conducted, the quark coupling arises from $\alpha_{U^{\prime}}=g_{U^{\prime}}^{2} / 4 \pi$ which is the baryonic fine structure constant and $g_{U^{\prime}}$ is the coupling coefficient.

$U^{\prime}$ is reconstructed taking advantage of the $\eta \rightarrow \pi^{+} \pi^{-} \gamma$ where the $\pi^{ \pm}$pair is possibly decaying from $U^{\prime}$ and $\eta$ is decaying from $D^{*+} \rightarrow D^{0} \pi^{+}, D^{0} \rightarrow K_{S}^{0} \eta$ and due to kinematics it allows for suppression of the combinatorial background, therefore a clearer signal.
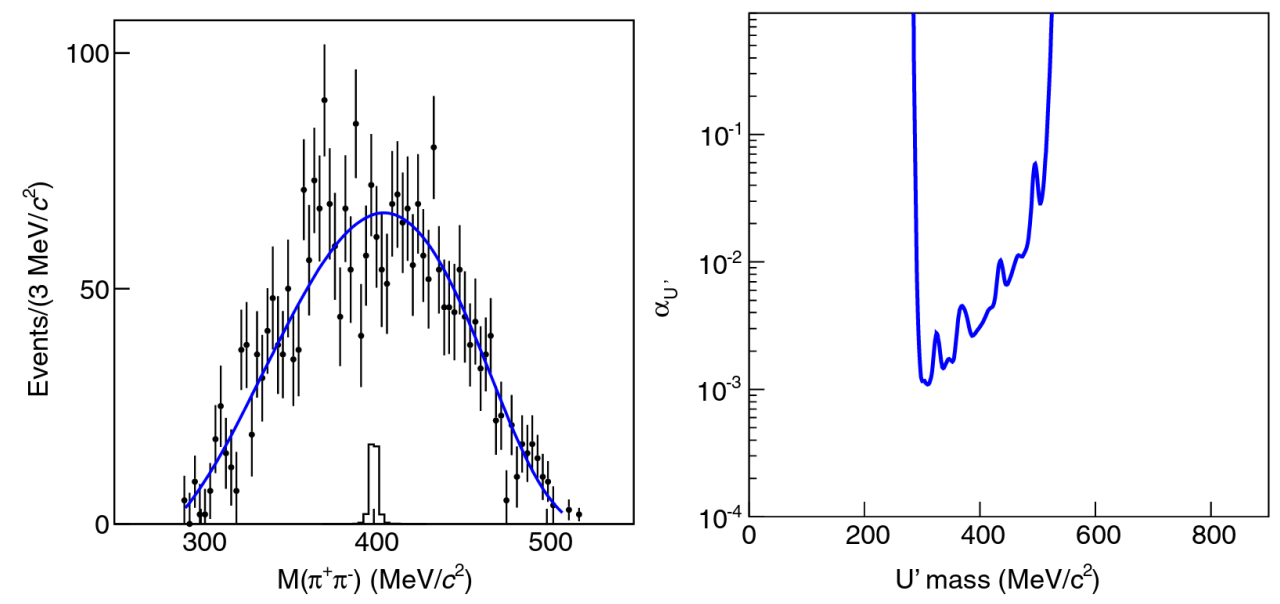

Figure 9: Invariant mass for the $\pi^{ \pm}$pair on the left data points with the differential decay rate fitted, solid curve, and a $U^{\prime}$ signal example at $400 \mathrm{MeV} / c^{2}$. On the right there is a exclusion zone plot of the fine structure constant $\alpha_{U^{\prime}}$ dependent with the $U^{\prime}$ mass $m_{U^{\prime}}$ [17]

By looking at the figure above, Fig. 9, it is possible to infer there is no enhancement in the $\pi^{ \pm}$pair mass distribution which is compatible of no $U^{\prime}$ found. Searches for a quark coupled dark gauge are also conducted in high energy colliders experiments, such as LHCb and CMS.

\subsection{Belle II}

Belle II upgrades are not yet completed, yet the expectation for the dark photon searches that have already been done at BES III and BaBar for Belle II are very optimistic, firstly the final full integrated luminosity will be far greater than that of BELLE, which was already a world record, as seen on Fig. 10. Apart from that it will have the single photon trigger present at BaBar, which BELLE did not have, allowing for cleaner dark photon ISR signals reconstructions.

\section{Summary}

Superficially covered a small spectrum of all the possible dark forces searches conducted in flavor experiments it is clear how diverse are the models for the dark sector and its portal interactions with the SM particles, and in some cases the analysis strategies might be similar. Other secluded gauge bosons that were not mentioned is the leptophilic dark $Z^{\prime}$ which was searched for in BaBar and is currently being search in BELLE data. Given the most recent upgrade completion from Belle II the prospects for dark photon searches and other are optimistic, if no signal is found many dark sector models might be constricted significantly. 


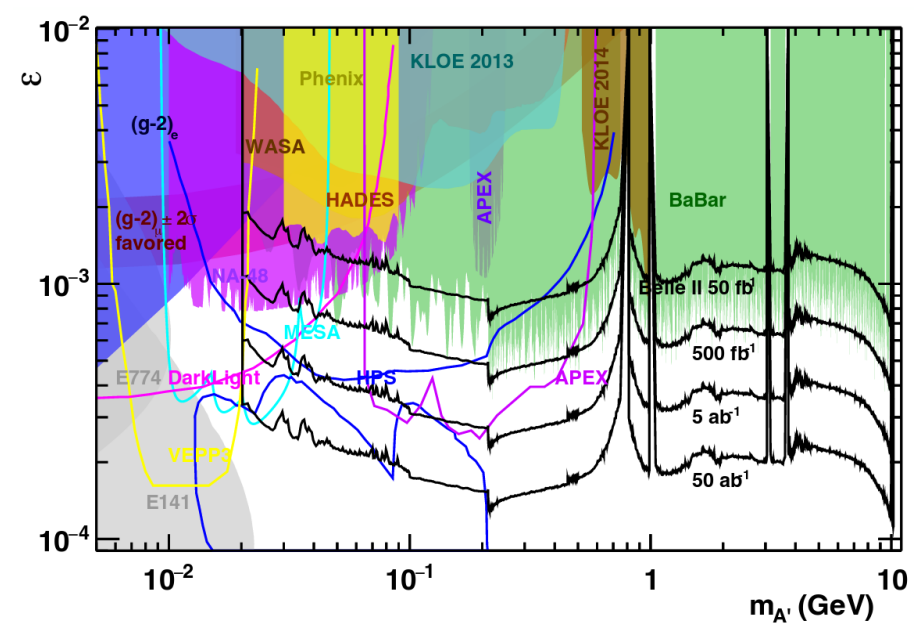

Figure 10: Dark photon kinematic mixing parameter by the dark photon candidate mass exclusion zones with respective integrated luminosities. [18]

\section{References}

[1] Stacy S. McCaugh, Federico Lelli and James M. Schombert, Radial Acceleration Relation in Rotationally Supported Galaxies Phys. Rev. Lett. 117, 201101 - 2016

[2] Wayne Hu and Scott Dodelson, Cosmic Microwave Background Anisotropies Ann. Rev. Astron. Astrophys. 40:171-216, 2002

[3] Richard Massey, Thomas Kitching and Johan Richard, The dark matter of gravitational lensing Rep. Prog. Phys. 73 (2010) 086901

[4] Leszek Roszkowski, Enrico Maria Sessolo, Sebastian Trojanowski, WIMP dark matter candidates and searches - current issues and future prospects arXiv:1707.06277 [hep-ph]

[5] Norman A. Graf, Michael E. Peskin, Jonathan L. Rosner (editors), Planning the Future of U.S. Particle Physics The Snowmass 2013 Proceedings FERMILAB-CONF-13-648, SLAC-PUB-15960. Organized by The Division of Particles and Fields of the American Physical Society

[6] Louis E. Strigari, Neutrino Coherent Scattering Rates at Direct Dark Matter Detectors SLAC-PUB-15817 arXiv:0903.3630

[7] M.Aguillar et al. (AMS Collaboration) First Result from the Alpha Magnetic Spectrometer on the International Space Station: Precision Measurement of the Positron Fraction in Primary Cosmic Rays of 0.5-350GeV Phys. Rev. Lett. 110, 141102 - 2013

[8] Jim Alexander, Marco Battaglieri et al. Dark Sectors 2016 Workshop: Community Report arXiv:1608.08632[hep-ph]

[9] John F. Cherry, Alexander Friedland, Ian M. Shoemaker, Neutrino Portal Dark Matter: From Dwarf Galaxies to Ice Cube arXiv:1411.1071v1[hep-ph]

[10] Xiao-Gang He and Jusak Tandean, Low-mass dark-matter hint from CDMS II, Higgs boson at the LHC, and darkon models Phys. Rev. D 88, 013020 - 2013

[11] P.Sikivie, Experimental Tests of the "Invisible" Axion Phys. Rev. Lett. 51, 1415 - 1983 
[12] Kunio Kaneta, Hye-Sung Lee, Seokhoon Yun, Portal Connecting Dark Photons and Axioms Phys. Rev. Lett. 118, 101802 - 2017

[13] M. Ablikim, Z.H.An, et al. Design and construction of the BES III detector Nuc. Ins. and Meth. A, 614 (3) 2010, p.345

[14] M. Ablikim, M. N. Achasov and the BES III Collaboration, Dark Photon Search in the Mass Range Between 1.5 and $3.4 \mathrm{GeV} / \mathrm{c}^{2}$ arXiv:1705.04265v1 [hep-ex]

[15] Maxim Pospelov, Secluded U(1) below the weak scale Phys. Rev. D 80, 095002 - 2009

[16] Ed. A.J. Bevan, B. Golob, Th. Mannel, S. Prell, and B.D. Yabsley, The Physics of B Factories Eur.Phys. J. C74 (2014) 3026, SLAC-PUB-15968, KEK Preprint 2014-3

[17] E. Won et al. (Belle Collaboration) Search for a dark vector gauge boson decaying to $\pi^{+} \pi^{-}$using $\eta \rightarrow \pi^{+} \pi^{-} \gamma$ decays Phys. Rev. D 94, 092006 - 2016

[18] C. Hearty, Dark Photon measurements at Belle II at Dark Sectors Workshop, SLAC https://indico.cern.ch/event/507783/contributions/2151644/ attachments/1265800/1873678/Dark_photons_Belle_II_Hearty.pdf (2016) 